\title{
PRECISE POSITIONING OF DIAMAGNETICALLY LEVITATED MICROROBOT
}

\author{
Anil Demircali, ${ }^{1}$ Huseyin Uvet, ${ }^{2}$ Yusuf Kahraman, ${ }^{3}$ Tunc Kose, ${ }^{4}$ \\ Serhat Sisu, ${ }^{5}$ Kadir Erkan ${ }^{6}$
}

\begin{abstract}
In this article, we present a microrobot manipulation technique with high precision positional ability to move in a fluid environment with diamagnetic levitation. Untethered manipulation of microrobots by means of externally applied magnetic forces has been emerging as a promising field of research, particularly due to its potential for medical and biological applications. The decreased size of the robots makes them suitable for both in vitro applications such as sorting, moving, filtering micro particles (e.g. cells) within lab-on-a-chip platforms and in vivo applications such as minimallyinvasive surgeries or targeted drug delivery inside a human body. Precise (nano) positioning of the levitated microrobot on the pyrolytic graphite is demonstrated in the liquid. Positioning is achieved by the movement of a "lifter" magnet on the sensitive microstage. The suspended microrobot successfully tracked the identified roots. Our study is about controlling the microrobot suspended on the pyrolytic graphite with nano-precision via fixed lifting magnets. The purpose of the presented method is to eliminate the friction force between the surface of the substrate and the microrobot. Thus, high accuracy motion can be achieved.
\end{abstract}

UDC Classification: 606 DOI: http://dx.doi.org/10.12955/cbup.v5.1073

Keywords: Microrobots, Magnetic Levitation.

\section{Introduction}

Microrobots are used in biological and medical applications for a number of purposes such as drug delivery, biopsy, marking, cell manipulation, microparticle transport, etc., with minimal damage to the desired site (Mooney et al., 2014; Arai et al., 2012; Arai et al., 2010; Cui et al., 2011; Abbott et al.; 2010). In the microfluidic environment, principles of physics change with decreasing dimensions. The change in the number of Re (Reynolds Number) indicates that the environment has laminar or turbulent flow characteristics. In the environment with laminar flow characterization, micro-objects move in the water to transport the viscous medium character (Nelseon et al., 2013). Under the mentioned conditions, microfluidic channels are seen in different structures from microrobots for control of the intended processes. Microrobotic applications can benefit from factors such as optical tweezers, thermal gradients, electrostatic forces, di-electrophoresis forces, chemical concentration differences (Ohta et al., 2012; Nelson et al., 2015). The use of microrobots in such applications is more advantageous than other applications when considering the effect on ambient conditions, the force to be applied and the precision of motion (Arai et al., 2012).

Recent studies show that Metin Sitti and Arai use magnetic levitation and acoustic levitation, respectively. These studies have controlled microrobots in the fluid environment by cutting off the contact with the surface. In both systems, the forces applied to retrieve position information and data collected from the sensors must change interactively. For this reason, ultrasonic vibrations affecting the positioning of microrobots are still present in the environment, and as a result, vibrations affect the objects in the environment. At this point, it can be seen that the last trend of studies to increase positioning accuracy and efficiency of applied force is based on levitation. Current levitation procedures require continuous energy consumption and feedback (Arai et al., 2016; Sitti el al, 2015). In the course of the work, it shows that microrobot systems are difficult to position precisely.

By cutting the surface contact; Diamagnetic levitation technique is an important method for precise positioning. With a permanent magnet to be positioned on the diamagnetic material, the microrobot can be levitated without the need for an active control mechanism. The diamagnetically levitated microrobot application was originally developed by Ron Pelrine et al. (Pelrine et al., 2012).

\footnotetext{
${ }^{1}$ Yildiz Technical University, Istanbul, Turkey, demircalianil@gmail.com

${ }^{2}$ Yildiz Technical University, Istanbul, Turkey, huvet@yildiz.edu.tr

${ }^{3}$ Yildiz Technical University, Istanbul, Turkey, yus_kah@ @otmail.com

${ }^{4}$ Yildiz Technical University, Istanbul, Turkey

${ }^{5}$ Yildiz Technical University, Istanbul, Turkey

${ }^{6}$ Yildiz Technical University, Istanbul, Turkey, kerkan@yildiz.edu.tr
} 
For the first time in the literature, we present a new method of using diamagnetic levitation in microrobotics in liquid media. Using this method, the theoretical background of a robotic work that can do 3-dimensional nano-positioning was established and confirmed by experimental results. In this way, innovative microrobotic systems can be developed that can integrate with lab-on-a-chip applications in a liquid environment.

\section{System Modelling}

Using the diamagnetic force, precise levitation and contactless manipulation of the microrobot arm can be performed (Pelrine et al., 2012; Chen, Zhou, Meng, 2008; Kustler, 2007). Movement limits within the fluid can be calculated by determining the system dynamics of the robot. According to the mathematical model of the system, the stability point of the robot can be determined in the liquid. The parameters used in the system model are given in Table-1. In Figure 2, the forces acting on the robotic arm z-axis are shown on the schematic given system. In addition, the forces resulting from moving the lifting magnet on the $\mathrm{x}$-axis are also expressed by the $\mathrm{x}$-axis components.

\begin{tabular}{|c|l|c|l|}
\hline \multicolumn{1}{|l|}{ Table 1: System Model Parameters } \\
\hline Parameters & \multicolumn{1}{|c|}{ Explanation } & Parameters & \multicolumn{1}{c|}{ Explanation } \\
\hline $\boldsymbol{F}_{\boldsymbol{B}}$ & Buoyant force & $\boldsymbol{V}_{\boldsymbol{p}}$ & Volume of a Particle \\
\hline $\boldsymbol{F}_{\boldsymbol{g}}$ & Diamagnetic force & $\boldsymbol{m}_{\boldsymbol{r}}$ & Robot mass \\
\hline $\boldsymbol{F}_{\boldsymbol{D}}$ & Drag force & $\boldsymbol{g}$ & Gravitational acceleration \\
\hline $\boldsymbol{F}_{\boldsymbol{m}}$ & Magnetic force & $\boldsymbol{V}_{\boldsymbol{r}}$ & Robot volume \\
\hline $\boldsymbol{F}_{\boldsymbol{r}}$ & Gravitational force & $\boldsymbol{\rho}_{\boldsymbol{f}}$ & Fluid density \\
\hline $\boldsymbol{B}$ & Magnetic flux density & $\boldsymbol{\rho}_{\boldsymbol{r}}$ & Robot density \\
\hline $\boldsymbol{H}$ & Magnetic field strength & $\boldsymbol{c}_{\boldsymbol{d}}$ & Drag coefficient \\
\hline $\boldsymbol{M}$ & Magnetization vector & $\boldsymbol{A}$ & Cross sectional area \\
\hline $\boldsymbol{\chi}$ & Magnetic insulation coefficient & $\boldsymbol{v}$ & Velocity \\
\hline $\boldsymbol{\mu}_{\boldsymbol{r}}$ & Relative permeability & \\
\hline Source: Author & & & \\
\hline
\end{tabular}

Figure 2: In the modelling, an acrylic container with DI water was used. Pyrolytic graphite was placed on the surface of the vessel and a robot arm was placed on the surface of the graphite. The ring magnet (called lifting-magnet) has 3 Degrees of Freedom (DOF) motion in the X-Y-Z axes. In our model, the forces acting on the microrobotic arm are given as follows.

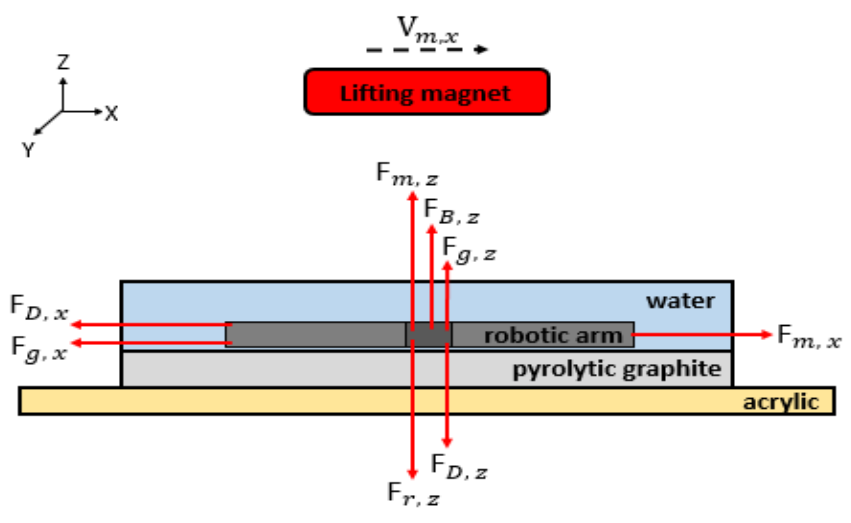

Source: Author

The forces shown in Figure 2 can be expressed as (1-3).

$$
\begin{aligned}
& F_{r}=m_{r} g \\
& F_{B}=V_{r}\left(\rho_{r}-\rho_{f}\right) g
\end{aligned}
$$




$$
F_{D}=\frac{1}{2} c_{d} \rho_{f} A v^{2}
$$

According to (1-3) equations, the dynamic model can be expressed by (4) for $\mathrm{z}$ axis.

$\ddot{z}=\frac{1}{2 m_{r}} c_{d} \rho_{f} A_{z} v_{z}\left|v_{z}\right|+\frac{V_{r} g}{m_{r}}\left(\rho_{r}-\rho_{f}\right)-g+\frac{\left(F_{m, z}+F_{g, z}\right)}{m_{r}}$

$v^{2}$ for the equation given in (3), is shown as $v|v|$ in the equation (4-6). Such an expression has been used for the positive and negative positional effects in the axes to be observed in the drag force expression. In the determination of $F_{m}$ and $F_{g}$ forces not shown in the equation set, basic physics is used first. The reason for this is the correct determination of the boundary conditions of the simulation in the analysis to be done in $\mathrm{COMSOL}^{\circledR}$. Thus, the magnetic force acting on the microrobot arm for the permanent magnet is theoretically expressed as follows.

$$
\begin{aligned}
& F_{m}=\frac{\mu_{r}-1}{2 \mu_{0} \mu_{r}} \iiint_{V} \nabla B^{2} d v \\
& F_{m}=\frac{\mu_{r}-1}{2 \mu_{0} \mu_{r}} \iint_{S} B^{2} d s
\end{aligned}
$$

Considering the Thomson's formulation for the microrobot arm,

$F_{m}=\frac{V_{p} \chi}{2 \mu_{0} \mu_{r}}(B \nabla) B$

the force between the lifting magnet and the robot arm is formulated. There are boundary conditions such as $\nabla B^{2}>0$ and $\left.\mu_{r}-1\right)<0$ to ensure stability at the equilibrium point. From this equation, a magnetic field gradient is needed so that a linear force can be generated on the robot. The position of the microrobot can be controlled by positioning the gradient of the magnetic field within it relative to the microrobot. In calculating the diamagnetic force, which is another magnetic force, calculation is made assuming that the material used is uniform. If the diamagnetic force components in $\mathrm{Z}$ direction is simplified according to Ostrogradsky's divergence law (Katz, 1979) as $F_{d i a, z}$,

$$
F_{d i a, z}=\left.\frac{\chi_{d i a}}{\mu_{0}} \oiint_{s}|B|\right|^{2} n_{z} d s
$$

where $n_{x}, n_{y}, n_{z}$ are the surface normal vector component of the diamagnetic material in $\mathrm{x}, \mathrm{y}, \mathrm{z}$ direction respectively; ds is the surface area unit of the diamagnetic material. The force between the diamagnetic material and the floating magnet can be calculated through equations (8). In addition, the net force (9) can be expressed by a single parameter to simplify the sum of the magnetic forces acting on the microrobot.

$$
F_{\text {net }}=F_{m}+F_{g}
$$

After the mathematical model is formed, the relevant parameters have to be calculated except $c_{d}$ and $F_{n e t}$. Numerical analysis is necessary to calculate them. Because the drag coefficient, $c_{d}$, varies depending on the stress, which is also depending on the velocity of the robot. Thus, (3) Number of equation is reshaped and $c_{d}$ left alone,

$c_{d}=\frac{2 F_{D}}{\rho_{f} A v^{2}}$

$F_{D}$ shall be calculated to determine drag force coefficient. To do this, the FSI (fluid structure interface) module in time-dependent is used. Time-dependent analyzes allow the simulation conditions to vary with time. For this reason, instead of "stationary", "time-dependent" analysis method is used in drag force calculation. Moreover, $F_{n e t}$ can be calculated with the MNFC (magnetic field no 
currents) module when stationary analysis mode is applied according to distance between the microrobot and the magnetic materials as well.

\begin{tabular}{|c||c||c|}
\hline \multicolumn{2}{|l|}{ Table 2: Calculated parameters in equ. (4) } \\
\hline Parameters & Value & Unit \\
\hline $\boldsymbol{m}_{\boldsymbol{r}}$ & $2.929751 \times 10^{-6}$ & $\mathrm{~kg}$ \\
\hline $\boldsymbol{g}$ & 9.81 & $\mathrm{~m} / \mathrm{s}^{2}$ \\
\hline $\boldsymbol{\rho}_{\boldsymbol{r}}$ & 1798.374 & $\mathrm{~kg} / \mathrm{m}^{3}$ \\
\hline $\boldsymbol{\rho}_{\boldsymbol{f}}$ & 998.2071 & $\mathrm{~kg} / \mathrm{m}^{3}$ \\
\hline $\boldsymbol{A}_{\boldsymbol{x}}$ & $1.229066 \times 10^{-6}$ & $\mathrm{~m}^{2}$ \\
\hline $\boldsymbol{A}_{\boldsymbol{y}}$ & $1.229066 \times 10^{-6}$ & $\mathrm{~m}^{2}$ \\
\hline $\boldsymbol{A}_{\boldsymbol{z}}$ & $8.15402 \times 10^{-6}$ & $\mathrm{~m}^{2}$ \\
\hline $\boldsymbol{V}_{\boldsymbol{r}}$ & $1.61911 \times 10^{-9}$ & $\mathrm{~m}^{3}$ \\
\hline \multicolumn{2}{|}{} \\
\hline Source: Author
\end{tabular}

After the theoretical values have been determined in Table 2, the relevant experimental setup is shown for the calculation of $c_{d}$ and $F_{n e t}$.

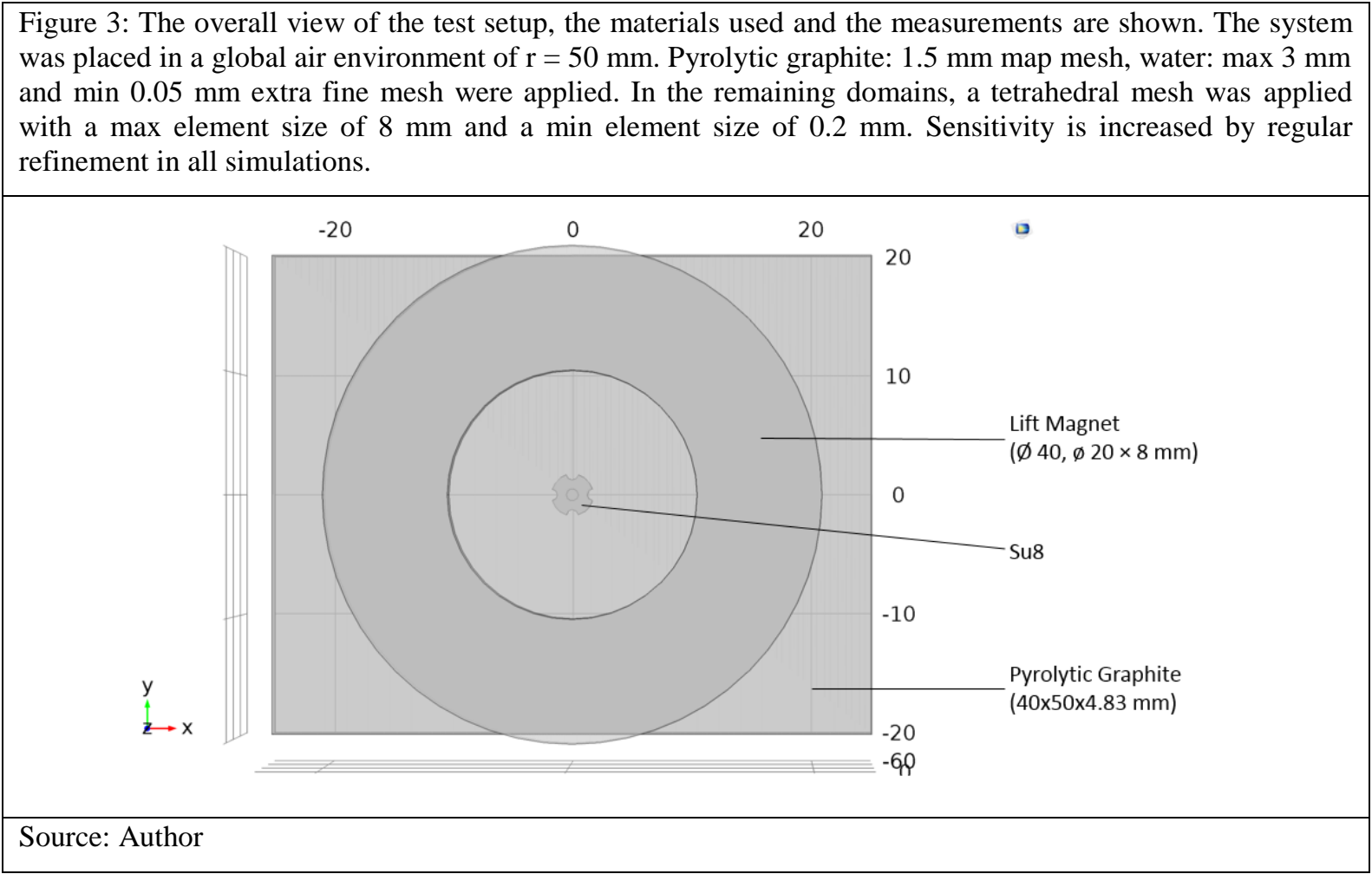

On the basis of the analysis given in Figure 3, the interaction of the robot arm with water was focused on and analysed. The microrobotic arm in the steady DI-water has been subjected to a certain axial movement, and the $c_{d}$ has been obtained due to the stretching on its surface. In the analysis performed, the stresses for the $-Z$ axis were calculated and the surfaces were selected. The robot arm in the container filled with water of dimensions $4 \times 4 \times 2 \mathrm{~mm}$ was moved in the $-Z$ direction. At the speed range of $0-6.83 \mathrm{~mm} / \mathrm{s}$, the stress between 16.6-348 $\mathrm{Pa}$ was observed on the robot surface. The analysis results are calculated as $c_{d}=7.681$ and $\max F_{D, z}=0.7815 \mu \mathrm{N}$.

Since the $\mathrm{F}_{\mathrm{D}}$ speed depends on the speed, the micro manipulator in which the lifting magnet is placed will vary according to the speed of the stage. Since the speed limits of the stage used in the experiments are in the range of $(-5-5) \mathrm{mm} / \mathrm{s}$, drag force and speed relation will show a parabolic variable behaviour in this range. According to the simulation results, it is calculated that the force generated for the maximum stage speed of $5 \mathrm{~mm} / \mathrm{s}$ is $0.781 \mu \mathrm{N}$. 
The other forces are fixed, except for the magnetic forces acting on the robot. For this reason, the determination of the magnetic forces after the drag force analysis, i.e., calculation of $F_{\text {net }}$, is necessary. As a result of the analysis, $F_{\text {net }}$ shows the point where the forces acting on the robot are zero. In the simulations, the lifting magnet heights corresponding to each zero value and levitation height for the robot were obtained. The analysis of the experimental setup was solved using the COMSOL direct solver method to obtain more accurate results. The result of the analysis is calculated by using the Maxwell stress tensor and global matrix evaluation method, which effects the robot arm, and the results are the same. The force obtained in the $\mathrm{Z}$ direction were taken with a $3.31 \%$ error by surface fitting is shown in Figure 4.

Figure 4: According to the parabolic surface graph obtained from the analysis made, when the "Lift Magnet
Distance" indicated by $\mathrm{Y}$ axis and the "Levitation Height" indicated by $\mathrm{X}$ axis increase, the net force
generated on the microrobot is reduced. Max: $51.89 \mu \mathrm{N}$ and min: $8.11 \mu \mathrm{N}$ force values were calculated.

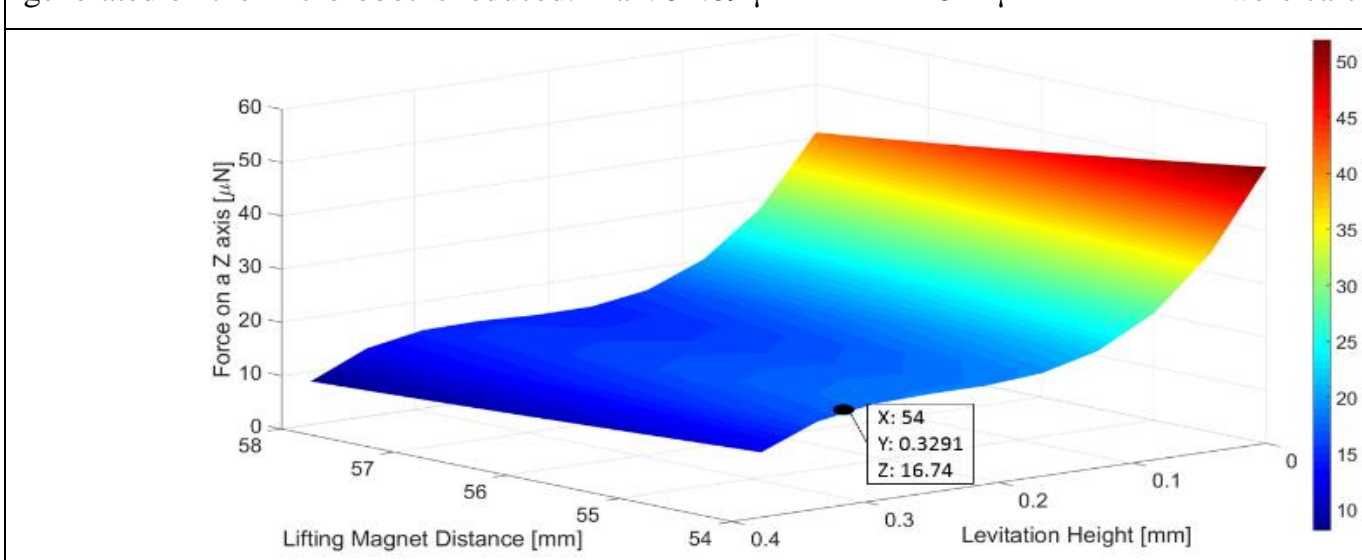

Source: Author

Along with the drag force obtained, the net force, lift force on the z-axis and microrobot mass act on the microrobot at the same time. Related forces;

$F_{T}=F_{n e t, z}-F_{D, z}+F_{B, z}-F_{r, z}$

\begin{tabular}{|c||c||c|}
\hline \multicolumn{3}{|c|}{ Table 3: Forces action on Microrobot z-axis } \\
\hline Forces & Value & Unit \\
\hline $\boldsymbol{F}_{\boldsymbol{D}, \boldsymbol{Z}}$ & 0.781 & $\mu N$ \\
\hline $\boldsymbol{F}_{\boldsymbol{B}, \boldsymbol{Z}}$ & 12.788 & $\mu N$ \\
\hline $\boldsymbol{F}_{\boldsymbol{r}, \boldsymbol{Z}}$ & 28.741 & $\mu N$ \\
\hline Source: Author \\
\hline
\end{tabular}

and $F_{T}$ (total force) are shown as equation (15). In addition, the related force values are given in Table 3 and the required magnetic force is calculated as $16.7345 \mu \mathrm{N}$. The corresponding force value given in Figure 6 is shown as marked on the surface graph. The corresponding force value given in Figure 6 is shown as marked on the surface graph. As a result, the robot is separated from the stabilization point at a levitation height of $329.1 \mu \mathrm{m}$ according to the analysis. In addition, there is a flat profile with a linear slope at the levitation height: $0.1-0.3 \mathrm{~mm}$. The area outside the region concerned may be called the region of instability; Within this region, the microrobot arm can be stably operated.

\section{Experimental Results}

Implementation of experimental setup; It contains a microrobot arm positioned in a DI water container with pyrolytic graphite on its surface. The ring magnet (lifter-magnet) on which the magnetic force required for levitation is obtained is on the vessel. To achieve stable and micro-precision levitation, it is necessary to position the lifter magnet on the DI water tank parallel and rigid. In order to perform levitation and precise position control at the micro level, A PI Micro Stage (M-126.PD2 / 20x20x20 $\mathrm{mm}$ ) with 3-axis linear micro-movement sensitivity was placed on the $\mathrm{Z}$ axis. A manual micro-stage was used to position the DI water container parallel to the floor and to move the 3 axes when 
necessary. A nano-sensitive laser distance sensor (optoNCDT-ILD2300-50) is preferred for instant measurement of levitation height within the system.

The microrobot is made to levitate by the movement of the fiber magnet in the order of microns along the $\mathrm{Z}$ axis. It exhibits a levitation characteristic depending on the magnets and properties used within the system. The reason for using a ring-shaped lifter magnet is that it has a hollow structure required for laser measurement. Thanks to the gap in the middle of the ring structure (10 mm diameter), the laser is aligned with the microrobot arm in the vertical position. The PI Stage, which we designed through the system interface, was moved by $50 \mu \mathrm{m}$ on the $\mathrm{Z}$ axis, followed by $10 \mu \mathrm{m}$ steps as the first levitation occurred. When the microrobot is in the levitated state, the behavior of the fiber magnet in the motion range of about $6 \mathrm{~mm}$ is observed. The measurement made according to the distance between the different levitation heights and the fibroid magnet microrobot is given in Figure 6.

Figure 6: The experiment was observed by recording the position data with the laser distance sensor in each movement step. The microrobot has been shown to operate at a maximum stabilization level of $330 \mu \mathrm{m}$ in the current system. Subsequently, the resultant force acting on the microrobot was acting in the upward direction, indicating that the robot departed from the stable region and moved upwards under the influence of the magnetic force.

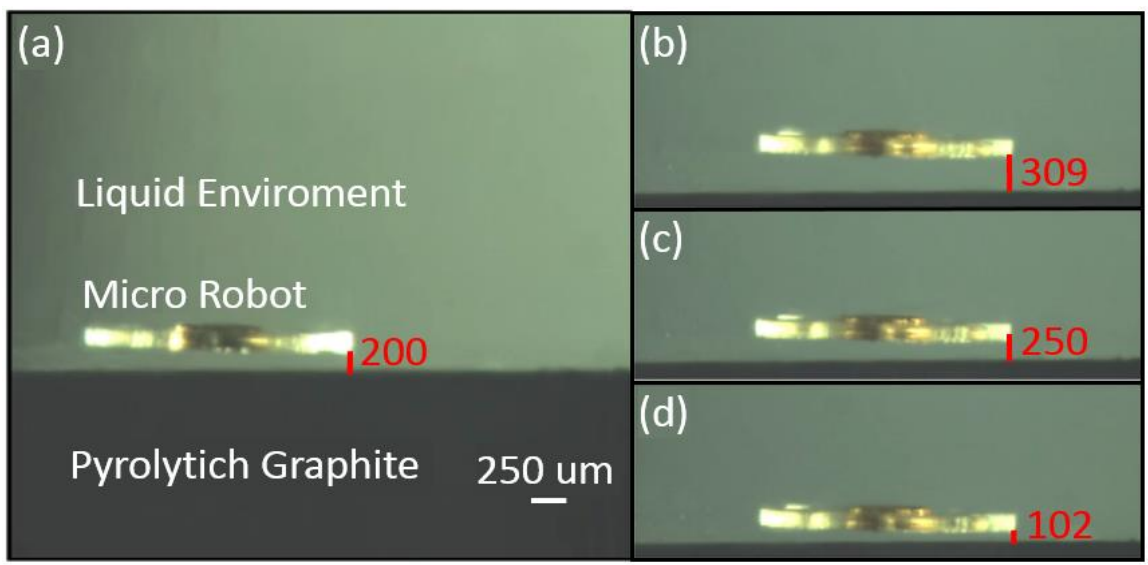

Source: Author

In addition, the change in the $6 \mathrm{~mm}$ between the lifter magnet and the graphite also affects the levitation height of the microrobot, as shown in Figure 7.

Figure 7: Prior to the critical region where the lifter magnet effect increased, the microrobot showed a levitation elevation with linear characteristics similar to the simulation results.

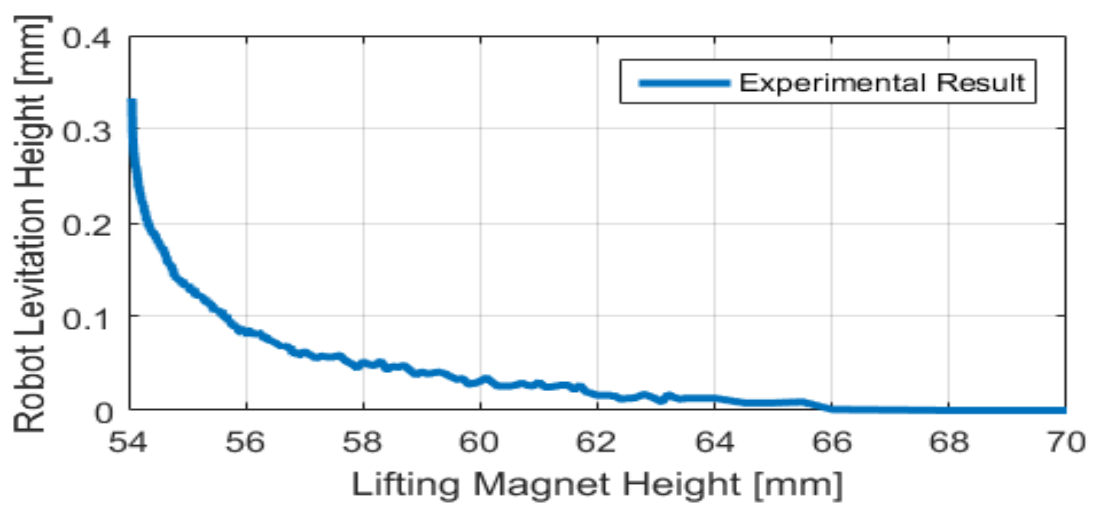

Source: Author

Considering the objects and motion field to be manipulated by the microrobot in lab-on-a-chip applications, the $333 \mu \mathrm{m}$ levitation height obtained from the experimental results is sufficient. In 
Figure 7, it is a characteristic of our existing system that we have designed and designed. The diamagnetically levitated method we have developed may vary according to the system requirements to be applied. The proposed manipulation method allows for the microrobot design and driving magnet properties to be modified to achieve specific levitation intervals for different applications.

\section{Conclusion}

With diamagnetic levitation, the microrobot has been removed from the surface to achieve high precision positioning. The method we have developed will provide new solutions for precise micronano size objects and microfluidic applications requiring high precision positioning.

In addition to horizontal and vertical axis positioning accuracy, applicability has been demonstrated by means of end elements. Precise positioning of the levitation height of the microrobot has also been proven by experimental findings. In this way, nano-precision levitation and position control are ensured so that the microrobot can control movement in the micro-fluidic channel, nano-level and 3 axes.

\section{Acknowledgement}

This project with Project No: 113E584 is supported by The Scientific and Technological Research Council of Turkey (TUBITAK).

\section{References}

Chen, J. Y., Zhou, J. B., \& Meng, G. (2008). Diamagnetic bearings for MEMS: Performance and stability analysis. Mechanics Research Communications, 35(8), 546-552.

Chung, S. E., Dong, X., \& Sitti, M. (2015). Three-dimensional heterogeneous assembly of coded microgels using an untethered mobile microgripper. Lab on a Chip, 15(7), 1667-1676.

Feng, L., Di, P., \& Arai, F. (2016). High-precision motion of magnetic microrobot with ultrasonic levitation for 3-D rotation of single oocyte. The International Journal of Robotics Research, 35(12), 1445-1458.

Feng, L., Ichikawa, A., Arai, F., \& Hagiwara, M. (2012, August). Continuous enucleation of bovine oocyte by microrobot with local flow distribution control. In Manipulation, Manufacturing and Measurement on the Nanoscale (3M-NANO), 2012 International Conference on (pp. 59-64). IEEE.

Fusco, S., Huang, H. W., Peyer, K. E., Peters, C., Häberli, M., Ulbers, A., ... \& Nelson, B. J. (2015). Shape-switching microrobots for medical applications: The influence of shape in drug delivery and locomotion. ACS applied materials \& interfaces, 7(12), 6803-6811.

Fusco, S., Sakar, M. S., Kennedy, S., Peters, C., Bottani, R., Starsich, F., ... \& Mooney, D. (2014). An Integrated icrorobotic Platform for On-Demand, Targeted Therapeutic Interventions. Advanced Materials, 26(6), 952-957.

Hagiwara, M., Kawahara, T., Yamanishi, Y., \& Arai, F. (2010). Driving method of microtool by horizontally arranged permanent magnets for single cell manipulation. Applied Physics Letters, 97(1), 013701.

Hu, W., Ishii, K. S., Fan, Q., \& Ohta, A. T. (2012). Hydrogel microrobots actuated by optically generated vapour bubbles. Lab on a Chip, 12(19), 3821-3826.

Katz, V. J. (1979). The history of Stokes' theorem. Mathematics Magazine, 52(3), 146-156.

Kustler, G. (2007). Diamagnetic levitation-historical milestones. Revue Roumaine Des Sciences Techniques Serie Electrotechnique Et Energetique, 52(3), 265.

Nelson, B. J., Kaliakatsos, I. K., \& Abbott, J. J. (2010). Microrobots for minimally invasive medicine. Annual review of biomedical engineering, 12, 55-85.

Pelrine, R., Wong-Foy, A., McCoy, B., Holeman, D., Mahoney, R., Myers, G., ... \& Low, T. (2012, May). Diamagnetically levitated robots: An approach to massively parallel robotic systems with unusual motion properties. In Robotics and Automation (ICRA), 2012 IEEE International Conference on (pp. 739-744). IEEE.

Peyer, K. E., Zhang, L., \& Nelson, B. J. (2013). Bio-inspired magnetic swimming microrobots for biomedical applications. Nanoscale, 5(4), 1259-1272.

Ye, Z., Duan, Z., \& Su, Y. (2015, February). Theoretic and numerical analysis of diamagnetic levitation and its experimental verification. In The International Conference on Photonics and Optical Engineering and the Annual West China Photonics Conference (icPOE 2014) (pp. 944907-944907). International Society for Optics and Photonics.

Zhang, T., Zhang, M., \& Cui, T. (2011, June). Microfluidic valves based on TiO 2 coating with tunable surface wettability between super hydrophilic and super hydrophobic. In Solid-State Sensors, Actuators and Microsystems Conference (TRANSDUCERS), 2011 16th International (pp. 306-309). IEEE. 